\title{
Estimation du progrès génétique réalisé chez le maïs grain en France entre 1950 et 1985
}

\author{
Maurice DERIEUX, Maurice DARRIGRAND (1), André GALLAIS ( $\left.{ }^{2}\right)$, Yves BARRIERE (3), Daniel \\ BLOC $\left({ }^{4}\right)$ \& Yves MONTALANT \\ I.N.R.A., Laboratoire d'Amélioration des Plantes, Centre de Recherches de Lille, Estrees-Mons, F 80200 \\ Peronne
}

\begin{abstract}
Trente-trois hybrides inscrits au catalogue entre 1950 et 1985 ont été étudiés pendant 3 ans $(1982,1983$ et 1984) à trois densités de peuplement. Les hybrides ont été répartis en 3 zones d'expérimentation en fonction de leur précocité (10 à 12 essais par zone).

Au cours de chaque décennie apparaissent des hybrides de plus en plus précoces. La liaison négative productivité $\times$ précocité impose une comparaison des rendements au même niveau de précocité. Pour cette raison chaque groupe de précocité a fait l'objet d'une séparation en 2 ou 3 séries de précocité différente.

Le progrès génétique moyen est de $0,8 \mathrm{q} / \mathrm{ha} / \mathrm{an}$ à comparer à un progrès agricole national moyen de $1,44 \mathrm{q} / \mathrm{ha} / \mathrm{an}$. Le progrès génétique représente donc $50 \mathrm{p}$. $100 \mathrm{du}$ progrès global. Le progrès est de $1 \mathrm{q} / \mathrm{ha} / \mathrm{an}$ pour les très précoces et les précoces, 0,6 pour les demi-précoces, 0,8 pour les tardifs.

L'amélioration de la résistance à la verse est très importante : le pourcentage de verse passe de $20 \mathrm{p} .100$ à 3,5 p. 100. De plus, la résistance à la verse facilite l'augmentation des densités de peuplement qui explique une partie du progrès sur le rendement. Le coefficient de régression du rendement sur la densité évolue de 0,08 pour les hybrides des années 1950 à 0,59 pour les hybrides des années 1980 .

Pour les hybrides très précoces, précoces et demi-précoces, l'amélioration porte sur la régularité du rendement et sur l'augmentation de la productivité. Cela signifie que les hybrides modernes sont mieux adaptés aux conditions difficiles (températures basses, verse, sécheresse, par exemple).

L'évolution des techniques culturales (densité de peuplement, fertilisation, irrigation, travail du sol) devrait contribuer à mieux utiliser le potentiel de production des cultivars.
\end{abstract}

Mots clés additionnels : Rendement, précocité, résistance à la verse, densité de peuplement.

Thirty-three maize hybrids grown in France during the last 30 years were compared over 3 years at 3 plant densities. The hybrids were split into 3 groups according to their earliness and studied in their areas of adaptation ( 3 sets of 10-12 trials). In each successive 10-year period, the hybrids became earlier. Since yield and earliness are well known to be related, comparisons had to be made at the same level of earliness. Accordingly, each geographic adaptation group was divided into 2 or 3 earliness subsets. From 1950 to 1980 the mean genetic gain was $0.08 \mathrm{t} / \mathrm{ha} /$ year to compare with the national mean agricultural gain of $0.144 \mathrm{t} / \mathrm{ha} /$ year over the same period. The genetic gain was thus about $50 \%$ of the total agricultural gain. The gain was $0.1 \mathrm{t} / \mathrm{ha} / \mathrm{year}$ for the very early and early hybrids, 0.06 for semi-early hybrids and 0.08 for late hybrids. The most important improvement was obtained for lodging resistance : percent lodging decreased from $20.2 \%$ to $3.5 \%$. Moreover this lodging resistance allowed an increase in planting density which explains a part of the yield gain. The regression of yield on density rose from 0.08 for the 1950 s hybrids to 0.59 for the 1980 s hybrids. For very early, early and semi-early hybrids, yield improvement resulted more from yield stability than from improvement of potential yield. In other words, modern hybrids are more adapted to hard conditions (e.g. low temperatures, drought).

Additional key words : Yield, earliness, lodging, plant density.

(1) AGPM, 122, boulevard Tourasse, F 64000 Pau.

(2) GIS MOULON, Ferme du Moulon, F 91190 Gif-sur-Yvette.

(3) I.N.R.A., Station d'Amélioration des Plantes, F 86600 Lusignan.

(4) AGPM, Station Expérimentale, Boigneville, F 97120 Maisse. 


\section{INTRODUCTION}

La culture moderne du maïs en France est récente, en particulier dans la moitié Nord du pays. Elle a connu depuis 30 ans une évolution spectaculaire :

- la surface cultivée pour le grain, passe de 300000 ha en 1950 à environ 1800000 ha en 1985. Le maïs ensilage, dont il ne sera pas question ici, occupe par ailleurs 1300000 ha. Ce développement de la culture existe dans toutes les zones, il s'y ajoute une extension importante vers le Nord;

- le rendement moyen national, d'environ $15 \mathrm{q} / \mathrm{ha}$ en 1950, atteint plus de $60 \mathrm{q} /$ ha en 1984. Cette augmentation résulte d'une part de l'amélioration génétique des variétés qui changent rapidement, d'autre part de l'évolution des techniques culturales. Le désherbage chimique, la mécanisation, l'utilisation de fumures plus copieuses, l'augmentation et la maîtrise des peuplements, l'irrigation se développent à partir de 1950 (CAuderon, 1983).

L'évolution variétale fait l'objet de cette étude. Pour tenter d'estimer le progrès dû à la génétique et à la sélection, nous avons étudié des hybrides d'époques différentes dans les mêmes conditions de milieu en utilisant des niveaux d'intensification différents (uniquement les densités de peuplement).

\section{MATÉRIEL ET MÉTHODES}

Trois zones d'expérimentation ont été définies en fonction des sommes de températures disponibles pendant la période de végétation du maïs (CAUDERON, 1958 ; BLOC et al., 1978) de façon à rassembler dans les essais des variétés de précocité comparable. La zone $\mathrm{AB}$ correspond aux variétés précoces et très précoces (indice FAO $<280$ ), la zone BC aux demiprécoces (indice FAO compris entre 270 et 400 ), la zone DE aux demi-tardives et tardives (indice FAO compris entre 400 et 700 ). Les essais ont été réalisés en 1982,1983 et 1984 . Au total entre 10 et 12 essais sont disponibles par zone (tabl. 1).

Une dizaine de variétés ont été mises en essai dans chaque zone. Leur choix est représentatif des variétés cultivées pendant une décennie. La liste des variétés est donnée dans le tableau 2 avec l'année d'inscription, le type d'hybride et la texture du grain.

Trois densités de peuplement ont été utilisées : 6,8 et 10 plantes par $\mathrm{m}^{2}$ pour le groupe précoce ; 5,7 et 8,5 pour les demi-précoces $; 4,5 ; 6$ et 7,5 pour les tardifs. Elles sont censées représenter les densités de peuplement utilisées au cours de la période considérée (1950-1985). Les autres facteurs culturaux sont homogènes et correspondent aux bonnes techniques culturales actuelles (désherbage, fertilisation...).

Les critères utilisés sont le rendement en grains ramené à 15 p. 100 d'eau, l'humidité du grain à la récolte qui indique la précocité de la variété, le nombre de pieds versés à la récolte qui confond verse racinaire et verse par pourriture des tiges. On a tenté de donner aux variétés un indice global de valeur agronomique en utilisant la méthode qui sert à l'inscription des variétés au catalogue officiel :

Indice de valeur agronomique $=$ Rendement en p. 100 des témoins $-2,5 \times$ (p. 100 eau - p. 100 eau témoin) $-0,75$ (p. 100 verse - p. 100 verse témoin).

Les semences des variétés qui ne sont plus commercialisées ont été produites par la station I.N.R.A. du maïs de Saint-Martin-de-Hinx.

Chaque essai représente un split-plot à 3 répétitions dont les densités constituent les grandes parcelles. La parcelle élémentaire est constituée de 4 lignes par variété dont les 2 lignes centrales seulement sont utili-

TABLEAU

Implantation des essais.

Trial locations.

\begin{tabular}{|c|c|c|c|}
\hline $\begin{array}{c}\text { Zone } \\
\text { d'expérimentation }\end{array}$ & Lieu & Années & Responsable \\
\hline A-B & $\begin{array}{l}\text { La Minière }(78) \\
\text { Boigneville }(91) \\
\text { La Verrière }(78) \\
\text { Péronne }(80) \\
\text { Le Moulon }(91) \\
\text { Lusignan }(86)^{*}\end{array}$ & $\begin{array}{l}1982-83-84 \\
1982-83-84 \\
1983-84 \\
1983-84 \\
1983 \\
1984\end{array}$ & $\begin{array}{l}\text { L. FELIX, I.N.R.A. } \\
\text { D. BLOC, AGPM } \\
\text { B. MILLE, I.N.R.A. } \\
\text { Y. MONTALANT, I.N.R.A. } \\
\text { J.-P. SAMPOUX, I.N.R.A. } \\
\text { Y. BARRIERE, I.N.R.A. }\end{array}$ \\
\hline $\mathrm{B}-\mathrm{C}$ & $\begin{array}{l}\text { Miossens }(64) \\
\text { Clermont-Ferrand (63) } \\
\text { Lusignan }(86) \\
\text { Colmar }(68) \\
\text { Gotheron }(26) * \\
\text { Guizerix }(65) \\
\text { Lème }(64)\end{array}$ & $\begin{array}{l}1982-83 \\
1982-83 \\
1983 \\
1984 \\
1983-84 \\
1983 \\
1984\end{array}$ & $\begin{array}{l}\text { M. Darrigrand, AGPM } \\
\text { M. PollaCSEK, I.N.R.A. } \\
\text { Y. BARRIERE, I.N.R.A. } \\
\text { C. MAGINIEAU, I.N.R.A. } \\
\text { C. Billot, I.N.R.A. } \\
\text { M. DARRIGRAND, AGPM } \\
\text { M. DARRIGRAND, AGPM }\end{array}$ \\
\hline D-E & $\begin{array}{l}\text { Montpellier (34) }{ }^{*} \\
\text { St-Martin-de-Hinx (40) } \\
\text { Miossens (64) } \\
\text { Sandreau (31) } \\
\text { Artagnan (65) }\end{array}$ & $\begin{array}{l}1982-83-84 \\
1982-83-84 \\
1982-83-84 \\
1982 \\
1983\end{array}$ & $\begin{array}{l}\text { F. KAAN, I.N.R.A. } \\
\text { A. PANOUILLE, I.N.R.A. } \\
\text { M. DARRIGRAND, AGPM } \\
\text { M. FARNARIE (OCCITANE DES SEMENCES) } \\
\text { M. DARRIGRAND, AGPM }\end{array}$ \\
\hline
\end{tabular}

* Essais irrigués. 
TABLEAU 2

Variétés mises en essai. Hybrids tested.

\begin{tabular}{|c|c|c|c|}
\hline Hybrides & $\begin{array}{c}\text { Année } \\
\text { d'inscription }\end{array}$ & $\begin{array}{c}\text { Type } \\
\text { d'hybride }\end{array}$ & $\begin{array}{l}\text { Texture } \\
\text { du grain }\end{array}$ \\
\hline \multicolumn{4}{|l|}{ ZONE $A-B$} \\
\hline $\begin{array}{l}\text { WISCONSIN } 240 \\
\text { WISCONSIN } 255\end{array}$ & $\begin{array}{l}1950 \\
1950\end{array}$ & $\begin{array}{l}\text { HD } \\
\text { HD }\end{array}$ & $\begin{array}{c}\text { denté (corné) } \\
\text { denté }\end{array}$ \\
\hline $\begin{array}{l}\text { I.N.R.A. } 200 \\
\text { I.N.R.A. } 258\end{array}$ & $\begin{array}{l}1957 \\
1958\end{array}$ & $\begin{array}{l}\text { HD } \\
\text { HD }\end{array}$ & $\begin{array}{l}\text { corné-denté } \\
\text { corné-denté }\end{array}$ \\
\hline $\begin{array}{c}\text { LG } 11 \\
\text { CP } 170\end{array}$ & $\begin{array}{l}1970 \\
1971\end{array}$ & $\begin{array}{l}\text { HTV } \\
\text { HTV }\end{array}$ & $\begin{array}{l}\text { corné-denté } \\
\text { corné }\end{array}$ \\
\hline $\begin{array}{c}\text { LIZA } \\
\text { LEADER } \\
\text { BRULOUIS I.N.R.A. } 180 \\
\text { DEA (1) } \\
\text { BROWNING I.N.R.A. }(150)\left({ }^{2}\right)\end{array}$ & $\begin{array}{l}1978 \\
1980 \\
1980 \\
1980 \\
1985\end{array}$ & $\begin{array}{l}\text { HTV } \\
\text { HTV } \\
\text { HTV } \\
\text { HS } \\
\text { HTV }\end{array}$ & $\begin{array}{l}\text { corné-denté } \\
\text { corné-denté } \\
\text { corné-denté } \\
\text { corné-denté } \\
\text { corné-denté }\end{array}$ \\
\hline \multicolumn{4}{|l|}{ ZONE B-C } \\
\hline $\begin{array}{l}\text { WISCONSIN } 355 \\
\text { WISCONSIN } 416\end{array}$ & $\begin{array}{l}1951 \\
1951\end{array}$ & $\begin{array}{l}\text { HD } \\
\text { HD }\end{array}$ & $\begin{array}{l}\text { denté } \\
\text { denté }\end{array}$ \\
\hline $\begin{array}{l}\text { I.N.R.A. } 260 \\
\text { I.N.R.A. } 420 \\
\text { I.N.R.A. } 430\end{array}$ & $\begin{array}{l}1961 \\
1961 \\
1962\end{array}$ & $\begin{array}{l}\text { HTV } \\
\text { HD } \\
\text { HD }\end{array}$ & $\begin{array}{l}\text { corné-denté } \\
\text { corné-denté } \\
\text { corné-denté }\end{array}$ \\
\hline $\begin{array}{c}\text { LG } 11 \\
\text { STAR } 304\end{array}$ & $\begin{array}{l}1970 \\
1973\end{array}$ & $\begin{array}{l}\text { HTV } \\
\text { HTV }\end{array}$ & $\begin{array}{l}\text { corné-denté } \\
\text { denté }\end{array}$ \\
\hline $\begin{array}{l}\text { LIZA } \\
\text { BRUEX } \\
\text { DEA }\end{array}$ & $\begin{array}{l}1978 \\
1980 \\
1980\end{array}$ & $\begin{array}{l}\text { HTV } \\
\text { HTV } \\
\text { HS }\end{array}$ & $\begin{array}{l}\text { corné-denté } \\
\text { corné-denté } \\
\text { corné-denté }\end{array}$ \\
\hline \multicolumn{4}{|l|}{ ZONE D-E } \\
\hline $\begin{array}{l}\text { WISCONSIN } 464 \\
\text { IOWA } 4417\end{array}$ & $\begin{array}{l}1951 \\
1951\end{array}$ & $\begin{array}{l}\text { HD } \\
\text { HD }\end{array}$ & $\begin{array}{l}\text { denté } \\
\text { denté }\end{array}$ \\
\hline $\begin{array}{l}\text { I.N.R.A. } 640 \\
\text { I.N.R.A. } 420 \\
\text { I.N.R.A. } 430 \\
\text { ILLINOIS } 3152 \\
\text { I.N.R.A. } 570\end{array}$ & $\begin{array}{l}1960 \\
1961 \\
1962 \\
1962 \\
1962\end{array}$ & $\begin{array}{l}\text { HD } \\
\text { HD } \\
\text { HD } \\
\text { HD } \\
\text { HD }\end{array}$ & $\begin{array}{l}\text { corné-denté } \\
\text { corné-denté } \\
\text { corné-denté } \\
\text { denté } \\
\text { corné-denté }\end{array}$ \\
\hline I.N.R.A. 508 & 1969 & HS & denté \\
\hline $\begin{array}{c}\text { ROC } \\
\text { MONSUR I.N.R.A. } 440 \\
\text { EVA } \\
\text { MOHICAN I.N.R.A. } 428\end{array}$ & $\begin{array}{l}1977 \\
1980 \\
1981 \\
1982\end{array}$ & $\begin{array}{l}\text { HS } \\
\text { HTV } \\
\text { HS } \\
\text { HTV }\end{array}$ & $\begin{array}{l}\text { denté } \\
\text { corné-denté } \\
\text { denté } \\
\text { corné-denté }\end{array}$ \\
\hline
\end{tabular}

(1) Seulement 6 essais sur 12.

(2) Seulement 10 essais sur 12.

sées. Certains essais ont été récoltés à la main, d'autres ont été récoltés mécaniquement en ramassant les épis à terre, enfin les derniers (environ 1/4) ont été récoltés mécaniquement sans ramasser les épis à terre.

\section{Analyses statistiques:}

Les analyses de variance ont été faites pour chaque essai puis pour l'ensemble des essais d'une zone avec le programme ANVARM de la bibliothèque AMANCE (BACHACOU et al., 1981). Ce programme calcule pour chaque niveau des facteurs (année, densité...) les moyennes corrigées du déséquilibre du plan d'expérience pour chacune des variables observées (rendement...), moyennes qui seront présentées ici. Quelle que soit la signification des effets simples et des interactions obtenues lors des calculs essai par essai, l'ensemble des essais a été utilisé dans l'établis- sement des moyennes pour prendre en compte l'ensemble de l'information disponible.

\section{RÉSULTATS}

\section{A. Zone A-B (hybrides précoces)}

Les caractéristiques agronomiques et les données regroupées par décennie d'origine des hybrides sont données dans le tableau 3. Pour rendre les comparaisons aussi pertinentes que possible, nous avons présenté les hybrides très précoces d'une part et les hybrides précoces d'autre part. Les hybrides BROWNING I.N.R.A. 150 et DEA ont été introduits en cours d'essai. Le premier présentait une précocité intéressante. Le second a pris une grande importance dans 
TABLEAU 3

Résultats agronomiques par hybride (moyenne de 3 densités) groupe précoce et très précoce. Agronomic results far each hybrid (mean of 3 densities) : very early and early group.

\begin{tabular}{|c|c|c|c|c|c|}
\hline \multirow{2}{*}{ Hybrides } & \multirow{2}{*}{$\begin{array}{l}\text { Décennie de } \\
\text { culture }\end{array}$} & \multicolumn{2}{|c|}{ Rendement } & \multirow{2}{*}{$\begin{array}{l}\text { Humidité du } \\
\text { grain à la } \\
\text { récolte }\end{array}$} & \multirow{2}{*}{$\begin{array}{l}\% \text { Verse } \\
\text { récolte }\end{array}$} \\
\hline & & $q / h a$ & $\%$ & & \\
\hline \multicolumn{6}{|l|}{ Série très précoce } \\
\hline W 240 & 1950 & 61,1 & 100 & 30,6 & 27,1 \\
\hline I.N.R.A. 200 & 1960 & 77,3 & 127 & 31,5 & 12,2 \\
\hline CP 170 & 1970 & 75,5 & 124 & 29,1 & 12,7 \\
\hline BRULOUIS & 1980 & 83,1 & 136 & 28,9 & 3,2 \\
\hline BROWNING & 1980 & 83,5 & 137 & 26,5 & 3,4 \\
\hline \multicolumn{6}{|l|}{ Série précoce } \\
\hline W 255 & 1950 & 69,0 & 100 & 30,9 & 18,8 \\
\hline I.N.R.A. 258 & 1960 & 79,9 & 116 & 33,1 & 8,4 \\
\hline LG 11 & 1970 & 88,2 & 128 & 32,4 & 6,4 \\
\hline LIZA & 1980 & 90,2 & 131 & 32,1 & 2,7 \\
\hline LEADER & 1980 & 89,0 & 129 & 31,5 & 9,0 \\
\hline DEA & 1980 & 96,6 & 140 & 34,6 & 1,0 \\
\hline F génotype & & $174^{* *}$ & & $330^{* *}$ & $75^{* *}$ \\
\hline \multicolumn{6}{|l|}{ Par décennie } \\
\hline & 1950 & 65,0 & 100 & 30,7 & 22,9 \\
\hline & 1960 & 78,6 & 121 & 32,3 & 10,3 \\
\hline & 1970 & 81,8 & 126 & 30,7 & 9,5 \\
\hline & 1980 & 88,4 & 136 & 30,7 & 3,9 \\
\hline
\end{tabular}

** Significatif au seuil 0,01 .

cette zone. Lors de la préparation de ce travail (1981), il était nouveau et semblait trop tardif pour figurer dans ces essais, en particulier dans les implantations les plus froides. Les résultats donnés ici sont les ajustements calculés à partir de 10 essais pour BROWNING, 6 essais pour DEA.

\section{Rendement}

Dans la série très précoce, le rendement augmente de $22,0 \mathrm{q} / \mathrm{ha}$. Le progrès le plus important a été apporté par I.N.R.A. 200 en 1957, mais le progrès est encore important avec les variétés récentes. Dans la série précoce, le rendement augmente de 27,6 q/ha, le progrès est continu avec I.N.R.A. 258, LG 11 et DEA.

$\mathrm{Si}$ l'on regroupe les variétés par décennie, on obtient un gain de $23,4 \mathrm{q} / \mathrm{ha}$ en 30 ans soit presque $0,8 \mathrm{q} / \mathrm{ha} / \mathrm{an}$.

La figure 1 présente la régression des variétés regroupées par décennie à la moyenne par essai, elle montre que l'amélioration génétique a augmenté la régularité du rendement, les écarts étant plus grands dans des conditions défavorables de milieu (faible rendement moyen de l'essai) que dans des conditions favorables (rendement élevé de l'essai).

2. Précocité (mesurée par la teneur en eau du grain à la récolte)

Le choix variétal conduit à une précocité légèrement améliorée de 1960 à 1970 et à 1980 (tabl. 3). Par conséquent, compte tenu de la régression négative rendement $\times$ précocité, les différences de rendement observées sont minimisées par l'effet précocité.
Par rapport aux variétés plus classiques, il faut noter l'apparition d'hybrides de plus en plus précoces : CP 170, BRULOUIS I.N.R.A. 180 et BROWNING I.N.R.A. 150.

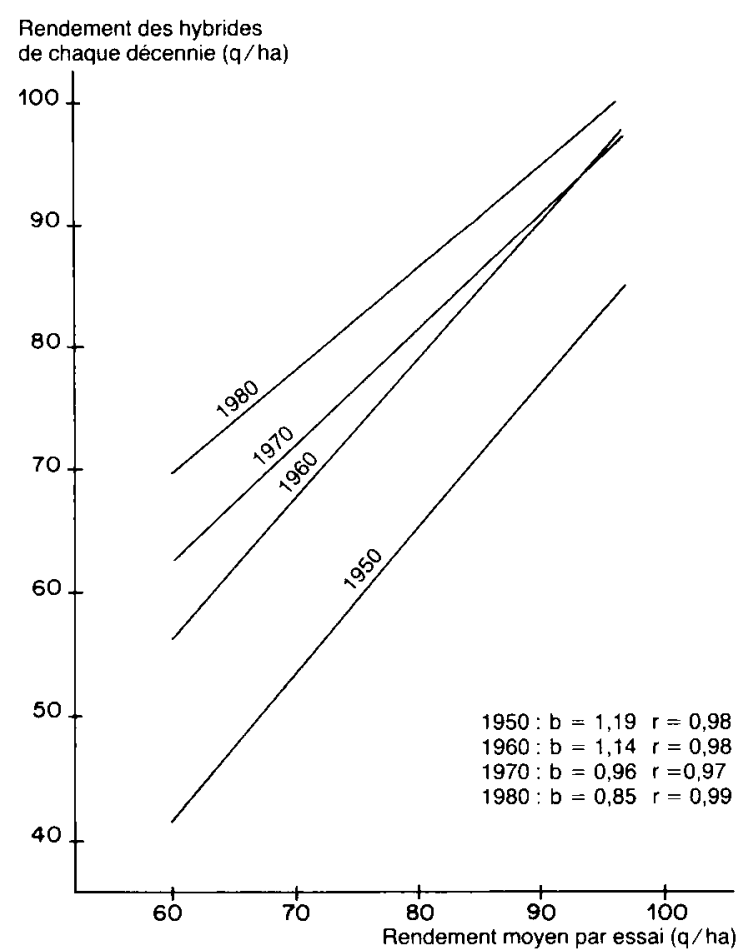

Figure 1

Relation par décennie entre le rendement des hybrides et la moyenne de chaque essai (hybrides précoces).

Yield of hybrids from each era related to the mean yield per trial (early hybrids). 


\section{Verse à la récolte}

La résistance à la verse est améliorée de façon très importante, puisque le pourcentage de verse est de 22,9 p. 100 pour les hybrides de 1950 et de 5,0 p. 100 pour les hybrides de 1980 .

Même les variétés très précoces (BRULOUIS et BROWNING) ont un très bon comportement à la verse.

\section{Effet des densités de peuplement}

\section{a) Rendement}

Le rendement augmente avec la densité. L'interaction variété $\times$ densité indique un comportement variable selon les hybrides, les moyennes sont données dans le tableau 4 avec le coefficient de régression par variété. L'effet densité est très important chez I.N.R.A. 200 et BROWNING I.N.R.A. 150 alors que les variétés anciennes $\mathrm{W} 240$ et $\mathrm{W} 255$ ont une réaction nulle ou légèrement négative.

L'estimation du progrès génétique doit se faire sur la densité optimale. On obtient alors un progrès de $24,1 \mathrm{q} / \mathrm{ha}$ dans la série très précoce et $25,5 \mathrm{q} / \mathrm{ha}$ dans la série précoce, globalement le progrès est donc de $1 \mathrm{q} / \mathrm{ha} / \mathrm{an}$.

\section{b) Précocité}

L'augmentation de la densité entraîne une légère diminution de la précocité. L'interaction variété $x$ densité n'est pas significative, ce qui montre que toutes les variétés réagissent de la même façon à l'augmentation des densités. c) Verse

L'effet densité n'est pas significatif, cependant les moyennes augmentent légèrement avec les densités. L'interaction densité $\times$ variété est significative au seuil de 5 p. 100. La variété LEADER tolère mal les fortes densités. A forte densité, les variétés les plus résistantes sont LIZA, BROWNING, BRULOUIS et LG 11.

\section{Conclusion pour le groupe précoce $(A-B)$}

Pour ce groupe, il faut donc retenir un gain de rendement de $1 \mathrm{q} / \mathrm{ha} / \mathrm{an}$, une amélioration très nette de la résistance à la verse et l'apparition d'hybrides de plus en plus précoces. Les indices de valeur agronomique atteignent 157 (BRULOUIS) et 169 (BROWNING) contre 100 pour W 240 chez les très précoces, 141 (LIZA) et 152 (DEA) contre 100 pour W $255 \mathrm{chez}$ les précoces.

\section{B. Zone B-C (hybrides demi-précoces)}

Les caractéristiques agronomiques par hybride sont présentées en une série demi-précoce et une série demitardive pour faciliter les comparaisons (tabl. 5). Les hybrides BRUEX et I.N.R.A. 420 jugés respectivement trop précoce et trop tardif pour le groupe n'ont pas été retenus dans les moyennes des hybrides regroupés par décennie d'obtention.

TABLEAU 4

Interaction variété $\times$ densité pour le rendement - hybrides précoces.

Variety $\times$ density interaction for yield - very early and early hybrids.

\begin{tabular}{|c|c|c|c|c|c|c|c|c|c|}
\hline \multirow{3}{*}{ Hybrides } & \multirow{3}{*}{$\begin{array}{l}\text { Décennie } \\
\text { de culture }\end{array}$} & \multirow{3}{*}{$\begin{array}{c}\text { Densité } 1 \\
60000 \\
\end{array}$} & \multirow{3}{*}{$\begin{array}{c}\text { Densite } 2 \\
80000\end{array}$} & \multirow{3}{*}{$\begin{array}{c}\text { Densité } 3 \\
100000\end{array}$} & \multirow{3}{*}{ Moyenne } & \multirow{3}{*}{$\begin{array}{l}\text { Coefficient } \\
\text { de régression } \\
\text { variété } \times \\
\text { densité }\end{array}$} & \multicolumn{3}{|c|}{ Densité optimale } \\
\hline & & & & & & & \multicolumn{2}{|c|}{ Rendement } & \multirow{2}{*}{$\begin{array}{l}\text { Indice de } \\
\text { valeur } \\
\text { agronomique }\end{array}$} \\
\hline & & & & & & & $q / h a$ & $\%$ & \\
\hline \multicolumn{10}{|l|}{ Série très précoce } \\
\hline W 240 & 1950 & 62,2 & 60,4 & 60,7 & 61,1 & $-0,04$ & 62,2 & 100 & 100 \\
\hline I.N.R.A. 200 & 1960 & 69,5 & 79,0 & 83,3 & 77,3 & 0,35 & 83,3 & 134 & 144 \\
\hline CP 170 & 1970 & 73,8 & 76,4 & 76,3 & 75,5 & 0,06 & 76,4 & 123 & 137 \\
\hline BRULOUIS & 1980 & 77,6 & 85,3 & 86,3 & 83,1 & 0,22 & 86,3 & 139 & 157 \\
\hline BROWNING & 1980 & 75,6 & 85,6 & 89,4 & 83,5 & 0,35 & 89,4 & 144 & 169 \\
\hline \multicolumn{10}{|l|}{ Série précoce } \\
\hline W 255 & 1950 & 69,3 & 69,1 & 68,5 & 69,0 & $-0,02$ & 69,3 & 100 & 100 \\
\hline I.N.R.A. 258 & 1960 & 75,8 & 83,0 & 80,8 & 79,9 & 0,13 & 83,0 & 120 & 120 \\
\hline LG 11 & 1970 & 81,7 & 90,0 & 92,8 & 88,2 & 0,28 & 92,8 & 134 & 141 \\
\hline LEADER & 1980 & 85,4 & 92,1 & 89,6 & 89,0 & 0,11 & 92,1 & 133 & 135 \\
\hline LIZA & 1980 & 83,9 & 91,8 & 94,8 & 90,2 & 0,27 & 94,8 & 137 & 141 \\
\hline DEA & 1980 & 88,6 & 92,9 & 102,4 & 96,6 & 0,35 & 102,4 & 148 & 152 \\
\hline
\end{tabular}

Moyennes par densité

\begin{tabular}{|c|c|c|c|c|c|c|}
\hline Rendement & 75,7 & 81,6 & 82,7 & 79,9 & $F$ densité : $93,1 * *$ & F variété $\times$ densité $: 5,34 * *$ \\
\hline$\%$ Matière sèclıe & 30,4 & 31,1 & 31,6 & 31,0 & $\mathrm{~F}$ densite $: 107,4^{* *}$ & $F$ variété $\times$ densité $: 1,22$ \\
\hline$\%$ Verse & 9,9 & 10,1 & 11,2 & 10,4 & F densité : & $F$ variété $\times$ densité $: 1,73^{*}$ \\
\hline
\end{tabular}

\footnotetext{
* Significatif au seuil 0,$05 ; * *$ Significatif au seuil $0,01$.
} 
TABLEAU 5

Résultats agronomiques par hybride (moyenne de 3 densités) groupe demi-précoce. Agronomic results for each hybrid (mean of 3 densities) : semi-early group.

\begin{tabular}{|c|c|c|c|c|c|}
\hline \multirow{2}{*}{ Hybrides } & \multirow{2}{*}{$\begin{array}{l}\text { Décennie de } \\
\text { culture }\end{array}$} & \multicolumn{2}{|c|}{ Rendement } & \multirow{2}{*}{$\begin{array}{l}\text { Humidité du } \\
\text { grain à la } \\
\text { récolte }\end{array}$} & \multirow{2}{*}{$\begin{array}{l}\% \text { Verse } \\
\text { récolte }\end{array}$} \\
\hline & & $\mathrm{g} / \mathrm{ha}$ & $\%$ & & \\
\hline \multicolumn{6}{|l|}{ Série demi-précoce } \\
\hline W 355 & 1950 & 70,6 & 100 & 26,2 & 18,3 \\
\hline I.N.R.A. 260 & 1960 & 79,7 & 113 & 26,1 & 6,6 \\
\hline LG 11 & 1970 & 74,6 & 106 & 25,1 & 5,9 \\
\hline LIZA & 1980 & 77,8 & 110 & 24,7 & 3,6 \\
\hline BRUEX (1) & 1980 & 73,1 & 104 & 23,4 & 9,5 \\
\hline \multicolumn{6}{|l|}{ Série demi-tardive } \\
\hline W 416 & 1950 & 75,1 & 100 & 27,1 & 16,6 \\
\hline I.N.R.A. $420(1)$ & 1960 & 82,5 & 110 & 31,3 & 7,7 \\
\hline I.N.R.A. 430 & 1960 & 85,0 & 113 & 28,0 & 11,4 \\
\hline STAR 304 & 1970 & 95,5 & 127 & 26,5 & 5,8 \\
\hline DEA & 1980 & 91,8 & 122 & 26,3 & 2,2 \\
\hline F génotype & & $98 * *$ & & $246 * *$ & $33 * *$ \\
\hline \multicolumn{6}{|l|}{ Par décennie } \\
\hline & 1950 & 72,8 & 100 & 26,6 & 17,4 \\
\hline & 1960 & 82,3 & 113 & 27,0 & 9,0 \\
\hline & 1970 & 85,0 & 118 & 25,8 & 5,8 \\
\hline & 1980 & 84,8 & 116 & 25,5 & 2,8 \\
\hline
\end{tabular}

(1) Hybrides non retenus dans le regroupement par décennie à cause d'une précocité trop éloignée de la moyenne du groupe.

** Significatif au seuil de 0,01 .

\section{Rendement}

Dans la série demi-précoce, le rendement augmente seulement de 7,2 q/ha. A noter que dès 1960, I.N.R.A. 260 procure un gain de rendement de $9,1 \mathrm{q} /$ ha et qu'ensuite il y a stagnation. Dans la série demi-tardive, le rendement augmente de $20,4 \mathrm{q} /$ ha. Là aussi le rendement maximal n'est pas obtenu avec une variété récente. Dans les regroupements par décennie d'obtention des hybrides, le rendement augmente de 12 quintaux en 30 ans c'est-à-dire $0,4 \mathrm{q} / \mathrm{ha} / \mathrm{an}$.

La régression des variétés représentant chaque décennie par rapport à la moyenne par essais (fig. 2) montre ici aussi une tendance à une meilleure régularité du rendement sauf pour les hybrides de 1970 pour lesquels l'hybride denté tardif STAR 304 introduit un comportement semblable aux hybrides dentés de 1950 .

\section{Précocité}

Les hybrides choisis pour représenter les années 1970 et 1980 sont légèrement plus précoces que les hybrides des années 1950 et 1960 (tabl. 5). Ici encore les différences de rendement observées sont minimisées par l'effet précocité. A noter que les humidités du grain sont en moyenne très basses (moyenne générale : 26,2 p. 100).

\section{Verse}

La résistance à la verse est améliorée de façon remarquable puisque le pourcentage de verse est de 17,4 pour les hybrides de 1950 et de 2,8 pour les hybrides de 1980 .

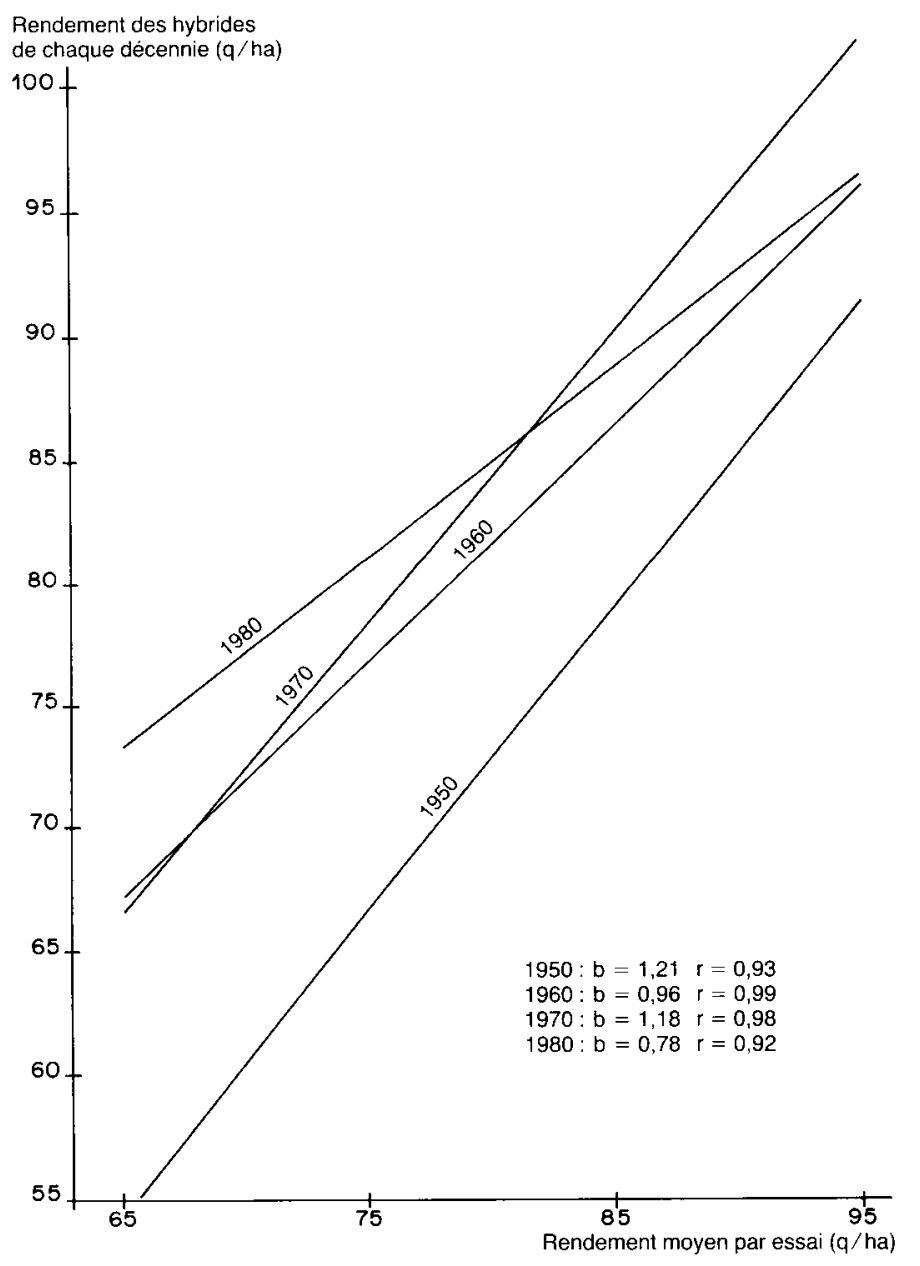

Figure 2

Relation par décennie entre le rendement des hybrides et la moyenne de chaque essai (hybrides demi-précoces).

Yield of hybrids from each era related to the mean yield per trial (semi-early hybrids). 


\section{Effet des densités de peuplement}

\section{a) Rendement}

Le rendement augmente avec la densité (tabl. 6).

L'interaction variété $\times$ densité est particulièrement intéressante. Les variétés anciennes ou de type denté tardif réagissent peu aux densités, alors que les variétés corné-dentées précoces réagissent davantage (tabl. 6). En se basant sur la densité optimale, on observe un gain de $12,9 \mathrm{q} /$ ha pour LIZA dans la série demi-précoce. Dans la série demi-tardive, DEA représente un progrès de $23 \mathrm{q} / \mathrm{ha}$. Globalement, le progrès est donc de $18 \mathrm{q} /$ ha sur 30 ans, soit $0,6 \mathrm{q} / \mathrm{ha} / \mathrm{an}$.

\section{b) Précocité}

La précocité diminue quand la densité augmente. Il n'y a pas d'interaction variété $\times$ densité.

\section{c) Verse à la récolte}

L'effet densité et l'interaction densité $\times$ variété ne sont pas significatifs. Quelles que soient les variétés, les fortes densités n'entraînent donc pas d'augmentation de la verse.

\section{Conclusion pour le groupe demi-précoce $(B-C)$}

Les indices de valeur agronomique calculés sur la densité optimale atteignent 132 (LIZA) contre 100 pour W 355 dans la série demi-précoce et 142 (DEA) contre 100 pour W 416 dans la série demi-tardive.

Pour ce groupe le progrès est donc de $0,6 \mathrm{q} / \mathrm{ha} / \mathrm{an}$, avec ici encore une amélioration très nette de la résistance à la verse.

\section{Zone D-E (variétés tardives)}

Pour rendre les comparaisons plus pertinentes, les caractéristiques agronomiques des hybrides sont présentées en 3 séries de précocité différente (tabl. 7). L'hybride ILLINOIS 3152, très tardif, n'est pas pris en compte dans la moyenne par décennie.

\section{Rendement}

Le rendement augmente de $26,3 \mathrm{q} /$ ha dans la série demi-tardive, $10,8 \mathrm{q} /$ ha dans la série tardive où il n'y a pas eu de progrès depuis 1970 (I.N.R.A. 508) et $24,9 \mathrm{q} / \mathrm{ha}$ dans la série très tardive. Le rendement par décennie augmente de $20 \mathrm{q} /$ ha en 30 ans soit un peu plus de $0,66 \mathrm{q} / \mathrm{ha} / \mathrm{an}$ (tabl. 7 ).

La régression des variétés regroupées par décennie par rapport à la moyenne par essai (fig. 3) montre de 1950 à 1960 et à 1970 , une tendance à une meilleure adaptation aux conditions favorables. Cette tendance se poursuit avec MOHICAN, alors que les autres hybrides de 1980 expriment au contraire une meilleure régularité de rendement. Il faut noter dans cette série l'existence d'un essai à MONTPELLIER en 1984 dont la moyenne est de $139 \mathrm{q} / \mathrm{ha}$.

\section{Précocité}

Le niveau d'humidité à la récolte est en moyenne assez bas. La précocité des variétés des années 80 est comparable à celle des années 50 , ce qui permet de ne pas biaiser les comparaisons de rendement.

TABLEAU 6

Interaction variété $\times$ densité pour le rendement - hybrides demi-précoces.

Variety $\times$ density interaction for yield - semi-early hybrids.

\begin{tabular}{|c|c|c|c|c|c|c|c|c|c|}
\hline \multirow{3}{*}{ Hybrides } & \multirow{3}{*}{$\begin{array}{l}\text { Décennie } \\
\text { de culture }\end{array}$} & \multirow{3}{*}{$\begin{array}{c}\text { Densité } 1 \\
60000\end{array}$} & \multirow{2}{*}{$\begin{array}{c}\text { Densité } 2 \\
80000\end{array}$} & \multirow{2}{*}{$\begin{array}{c}\text { Densite } 3 \\
100000\end{array}$} & \multirow{3}{*}{ Moyenne } & \multirow{3}{*}{$\begin{array}{l}\text { Coefficient } \\
\text { de régression } \\
\text { varièté } x \\
\text { densité }\end{array}$} & \multicolumn{3}{|c|}{ Densité optimale } \\
\hline & & & & & & & \multicolumn{2}{|c|}{ Rendement } & \multirow{2}{*}{$\begin{array}{l}\text { Indice de } \\
\text { valeur } \\
\text { agronomique }\end{array}$} \\
\hline & & & Rendem & nt $q / h a$ & & & $q /$ / $a$ & $\%$ & \\
\hline \multicolumn{10}{|c|}{ Série demi-précoce } \\
\hline W 355 & 1950 & 69,1 & 72,1 & 70,8 & 70,6 & 0,08 & 72,1 & 100 & 100 \\
\hline I.N.R.A. 260 & 1960 & 73,4 & 81,7 & 84,1 & 79,7 & 0,54 & 84,1 & 117 & 126 \\
\hline LG 11 & 1970 & 68,7 & 76,3 & 78,7 & 74,6 & 0,50 & 78,7 & 109 & 121 \\
\hline LIZA & 1980 & 69,0 & 79,5 & 85,0 & 77,8 & 0,80 & 85,0 & 118 & 132 \\
\hline BRUEX & 1980 & 66,6 & 75,0 & 77,5 & 73,1 & 0,55 & 77,5 & 107 & 120 \\
\hline \multicolumn{10}{|c|}{ Série demi-tardive } \\
\hline W 416 & 1950 & 73,8 & 75,5 & 76,0 & 75,1 & 0,11 & 76,0 & 100 & 100 \\
\hline I.N.R.A. 420 & 1960 & 81,7 & 83,3 & 82,5 & 82,5 & 0,04 & 83,3 & 110 & 104 \\
\hline I.N.R.A. 430 & 1960 & 80,9 & 86,2 & 88,0 & 85,0 & 0,35 & 88,0 & 116 & 111 \\
\hline STAR 304 & 1970 & 92,6 & 98,5 & 95,4 & 95,5 & 0,14 & 98,5 & 130 & 138 \\
\hline DEA & 1980 & 82,5 & 93,8 & 99,0 & 91,8 & 0,83 & 99,0 & 130 & 142 \\
\hline
\end{tabular}

Moyennes par densité

\begin{tabular}{|c|c|c|c|c|c|c|}
\hline Rendement & 75,8 & 82,2 & 83,7 & 80,5 & $\mathrm{~F}$ densité : $85,7^{* *}$ & F variete $\times$ densite $: 4,45 * *$ \\
\hline \% Matière sèche & 25,9 & 26,5 & 26,9 & 26,4 & $\mathbf{F}$ densite : $52,5 * *$ & F varicte $\times$ densite $: 1,05$ \\
\hline$\%$ Verse & 8,3 & 9,3 & 8,5 & 8,7 & F densiti : & F varicti $\times$ densite $: 1,53$ \\
\hline
\end{tabular}

** Significatif au seuil de 0,01 . 
TABLEAU 7

Résultats agronomiques par hybride (moyenne des 3 densités) hybrides tardifs. Agronomic results far each hybrid (mean of 3 densities) : late hybrids.

\begin{tabular}{|c|c|c|c|c|c|}
\hline \multirow{2}{*}{ Hybrides } & \multirow{2}{*}{$\begin{array}{l}\text { Décennie de } \\
\text { culture }\end{array}$} & \multicolumn{2}{|c|}{ Rendement } & \multirow{2}{*}{$\begin{array}{l}\text { Humidité du } \\
\text { grain à la } \\
\text { récolte }\end{array}$} & \multirow{2}{*}{$\begin{array}{l}\% \text { Verse } \\
\text { récolte }\end{array}$} \\
\hline & & $\mathrm{q} / \mathrm{ha}$ & $\%$ & & \\
\hline \multicolumn{6}{|l|}{ Série demi-tardive } \\
\hline W 464 & 1950 & 76,9 & 100 & 23,0 & 27,5 \\
\hline I.N.R.A. 430 & 1960 & 86,7 & 113 & 23,2 & 13,7 \\
\hline EVA & 1980 & 102,8 & 134 & 23,0 & 2,1 \\
\hline MOHICAN & 1980 & 103,2 & 134 & 23,1 & 6,8 \\
\hline \multicolumn{6}{|l|}{ Série tardive } \\
\hline IOWA 4417 & 1950 & 86,9 & 100 & 24,1 & 13,1 \\
\hline I.N.R.A. 570 & 1960 & 91,7 & 106 & 24,9 & 21,3 \\
\hline I.N.R.A. 508 & 1970 & 97,7 & 112 & 24,8 & 6,2 \\
\hline I.N.R.A. 440 & 1980 & 97,1 & 112 & 24,2 & 2,6 \\
\hline \multicolumn{6}{|l|}{ Série très tardive } \\
\hline IOWA 4417 & 1950 & 86,9 & 100 & 24,1 & 13,1 \\
\hline I.N.R.A. 640 & 1960 & 93,9 & 108 & 27,6 & 26,6 \\
\hline I.N.R.A. 420 & 1960 & 79,7 & 92 & 25,7 & 10,6 \\
\hline ILLINOIS $3152\left(^{1}\right)$ & 1960 & 92,9 & 107 & 29,0 & 10,5 \\
\hline ROC & 1980 & 104,6 & 120 & 25,9 & 4,1 \\
\hline F génotype & & $139 * *$ & & $419 * *$ & $71 * *$ \\
\hline \multicolumn{6}{|l|}{ Par décennie } \\
\hline & 1950 & 81,9 & 100 & 23,5 & 20,2 \\
\hline & 1960 & 87,9 & 107 & 25,3 & 18,0 \\
\hline & 1970 & 97,7 & 119 & 24,8 & 6,2 \\
\hline & 1980 & 101,9 & 124 & 24,0 & 3,9 \\
\hline
\end{tabular}

(1) Hybride non retenu dans le regroupement par décennie à cause d'une tardiveté excessive.

** Significatif au seuil de 0,01 .

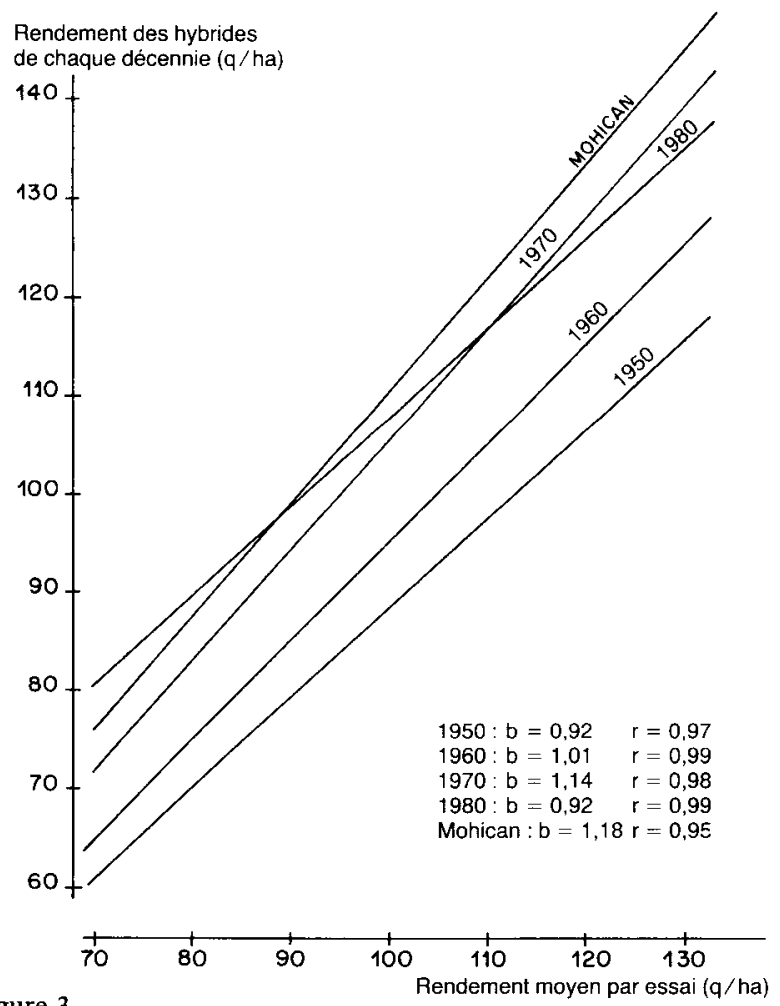

Figure 3

Relation par décennie entre le rendement des hybrides et la moyenne de chaque essai (hybrides tardifs).

Yield of hybrids from each era related to mean yield per trial (late hybrids).

\section{Verse à la récolte}

La résistance à la verse est améliorée de façon très importante puisque le pourcentage de verse est de
20,2 p. 100 pour les hybrides de 1950 et 3,9 p. 100 pour les hybrides de 1980 .

\section{Effet des densités de peuplements}

\section{a) Rendement}

Le rendement augmente avec la densité et l'interaction densité $\times$ variété indique un comportement différent selon les hybrides (tabl. 8). L'évolution au cours du temps se fait de façon très nette vers une adaptation aux fortes densités, sauf la variété prolifique MOHICAN qui réagit très peu à la densité de peuplement mais exprime un niveau de rendement élevé à densité faible. La capacité de cette variété à compenser un mauvais peuplement est donc manifeste.

En se référant à la densité optimale, le progrès est de $33,7 \mathrm{q} / \mathrm{ha}$ dans la série demi-tardive ; $15,9 \mathrm{q} / \mathrm{ha}$ dans la série tardive; $24,1 \mathrm{q} / \mathrm{ha}$ (par rapport à I. 4417) dans la série très tardive. Globalement, le progrès est donc de $24,6 \mathrm{q} / \mathrm{ha}$ en 30 ans soit un peu plus de $0,8 \mathrm{q} / \mathrm{ha} / \mathrm{an}$.

\section{b) Précocité}

La précocité diminue quand la densité augmente.

L'interaction variété $\times$ densité indique des réactions légèrement différentes selon les variétés.

\section{c) Verse}

La verse augmente avec la densité. L'interaction densité $\times$ variété indique que les variétés ont un comportement différent. Les variétés les plus résistantes à la verse à densité élevée sont ROC, I.N.R.A. 440 et EVA, pour lesquelles le pourcentage de verse n'augmente pas avec la densité. 
TABLEAU 8

Interaction variété $\times$ densité pour le rendement - hybrides tardifs.

Variety $\times$ density interaction for yield - late hybrids.

\begin{tabular}{|c|c|c|c|c|c|c|c|c|c|}
\hline \multirow{3}{*}{ Hybrides } & \multirow{3}{*}{$\begin{array}{l}\text { Décennie } \\
\text { de culture }\end{array}$} & \multirow{3}{*}{$\begin{array}{c}\text { Densité } 1 \\
60000\end{array}$} & \multirow{2}{*}{$\begin{array}{c}\text { Densité } 2 \\
80000\end{array}$} & \multirow{2}{*}{$\begin{array}{c}\text { Densité } 3 \\
100000\end{array}$} & \multirow{3}{*}{ Moyenne } & \multirow{3}{*}{$\begin{array}{c}\text { Coefficient } \\
\text { de régression } \\
\text { variété } \times \\
\text { densité }\end{array}$} & \multicolumn{3}{|c|}{ Densité optimale } \\
\hline & & & & & & & \multicolumn{2}{|c|}{ Rendement } & \multirow{2}{*}{$\begin{array}{l}\text { Indice de } \\
\text { valeur } \\
\text { agronomique }\end{array}$} \\
\hline & & & \multicolumn{2}{|c|}{ Rendement $\mathrm{q} / \mathrm{ha}$} & & & q/ha & $\%$ & \\
\hline \multicolumn{10}{|l|}{ Série demi-tardive } \\
\hline W 464 & 1950 & 74,9 & 78,2 & 77,7 & 76,9 & 0,09 & 78,2 & 100 & 100 \\
\hline I.N.R.A. 430 & 1960 & 80,4 & 89,5 & 90,1 & 86,7 & 0,32 & 90,1 & 115 & 122 \\
\hline EVA & 1980 & 90,6 & 105,9 & 111,9 & 102,8 & 0,71 & 111,9 & 134 & 151 \\
\hline MOHICAN & 1980 & 101,0 & 103,9 & 104,7 & 103,2 & 0,02 & 104,7 & 134 & 147 \\
\hline \multicolumn{10}{|l|}{ Série tardive } \\
\hline IOWA 4417 & 1950 & 82,7 & 88,0 & 90,2 & 86,9 & 0,25 & 90,2 & 100 & 100 \\
\hline I.N.R.A. 570 & 1960 & 87,2 & 92,1 & 95,9 & 91,7 & 0,29 & 95,9 & 106 & 93 \\
\hline I.N.R.A. 508 & 1970 & 89,5 & 99,8 & 103,7 & 97,7 & 0,47 & 103,7 & 115 & 116 \\
\hline I.N.R.A. 440 & 1980 & 85,8 & 99,4 & 106,1 & 97,1 & 0,68 & 106,1 & 118 & 125 \\
\hline \multicolumn{10}{|l|}{ Série très tardive } \\
\hline IOWA 4417 & 1950 & 82,7 & 88,0 & 90,2 & 86,9 & 0,25 & 90,2 & 100 & 100 \\
\hline I.N.R.A. 420 & 1960 & 75,6 & 80,1 & 83,6 & 79,7 & 0,27 & 83,6 & 93 & 91 \\
\hline I.N.R.A. 640 & 1960 & 90,7 & 95,9 & 95,1 & 93,9 & 0,15 & 95,9 & 106 & 89 \\
\hline ILLINOIS 3152 & 1960 & 88,1 & 95,3 & 95,4 & 92,9 & 0,24 & 95,4 & 103 & 90 \\
\hline ROC & 1980 & 92,7 & 106,7 & 114,3 & 104,6 & 0,72 & 114,3 & 127 & 129 \\
\hline \multicolumn{10}{|c|}{ Moyennes par densité } \\
\hline Rendement & & 86,6 & 97,6 & 97,3 & 92,9 & F densité : $224,4 * *$ & \multicolumn{3}{|c|}{ F variété $\times$ densité $: 7,44^{* *}$} \\
\hline$\%$ Matière sèche & & 24,6 & 24,9 & 25,2 & 24,9 & F densité : & \multicolumn{3}{|c|}{ F variété $\times$ densité $: 1,64^{*}$} \\
\hline$\%$ Verse & & 7,8 & 12,2 & 16,5 & 12,1 & F densité : $67,7 * *$ & \multicolumn{3}{|c|}{ F variété $\times$ densité $: 3,17^{* *}$} \\
\hline
\end{tabular}

* Significatif au seuil de 0,$05 ;{ }^{* *}$ Significatif au seuil de 0,01 .

\section{Conclusion pour le groupe tardif (D-E)}

Pour ce groupe, on retiendra donc un progrès de $0,8 \mathrm{q} / \mathrm{ha} / \mathrm{an}$ avec une forte amélioration de la résistance à la verse. Les indices de valeur agronomique atteignent 151 (EVA), 125 (I.N.R.A. 440), 129 (ROC) contre 100 pour les hybrides correspondants de 1950 .

\section{DISCUSSION ET CONCLUSION}

Il faut être prudent dans l'interprétation des résultats qui dépendent beaucoup des variétés mises en essai et du réseau d'essais. Les problèmes rencontrés dans notre étude qui résultent de ces choix sont :

\section{A. En zone AB}

Le grand nombre d'essais en région parisienne, la "percée " de DEA en cours d'expérimentation, alors que celui-ci ne figurait pas dans les hybrides initialement retenus.

\section{B. En zone BC}

Un réseau d'essais assez méridional, s'apparentant plutôt à une zone $\mathrm{CD}$, avantageant les hybrides dentés à fort potentiel. Dans cette zone figurent en effet des hybrides au comportement très différent en fonction des conditions de milieu (dentés et corné-dentés).

\section{En zone DE}

Il y a peu de problèmes de localisation des essais et de choix des hybrides. Dans ce groupe, les différences de précocité sont très importantes et une étude plus détaillée a été nécessaire.

Le rendement moyen du réseau précoce est de $81,2 \mathrm{q} / \mathrm{ha}$, celui du réseau demi-précoce : $80,6 \mathrm{q} / \mathrm{ha}$ et celui du réseau tardif : $92,4 \mathrm{q} / \mathrm{ha}$. Le réseau demiprécoce a sans doute souffert de facteurs limitants (peut-être alimentation hydrique) puisque les hybrides communs aux réseaux précoce et demi-précoce (LG 11, LIZA et DEA) donnent en moyenne $10 \mathrm{q} /$ ha de plus dans le réseau précoce. Par contre, les hybrides communs aux réseaux demi-précoce et tardif (I. 420 et $\mathrm{I}$. 430) donnent à peu près le même rendement dans les deux réseaux.

Il faut insister sur l'importance de la précocité dans nos conditions. La liaison très forte entre précocité et productivité fait que l'on ne peut comparer que la productivité des variétés de même précocité. Cela a été globalement réalisé grâce à la division en séries à l'intérieur des groupes de précocité. Dans le groupe demi-précoce, la comparaison de variétés cornédentées d'une part (DEA) et dentées d'autre part (STAR 304) est difficile, la précocité dépendant beaucoup du niveau d'humidité du grain (DERIEUX et al., 1986). Le type précoce est DEA, mais sa précocité peut être masquée, lorsque la récolte est faite à humidité faible du grain, par un grain qui se dessèche plus lentement que celui des types dentés (STAR 304). 
Dans nos essais, la précocité est exactement la même pour les 2 hybrides au niveau de 26,5 p. 100. De façon générale, les humidités du grain à la récolte sont plus basses que celles que l'on observe en culture, en particulier chez les précoces : LIZA, l'hybride le plus tardif du groupe est seulement à 32,1 p. 100 d'eau, BROWNING le plus précoce, est à 26,5 p. 100 .

Les relations négatives habituelles entre rendement, précocité et verse ne se retrouvent dans aucun des groupes de précocité, ce qui montre que ces caractères ont été améliorés simultanément (DERIEUX et al., 1986).

Conscients de ces limites, nous estimons que les progrès mesurés dans les différents groupes de précocité sont : $1 \mathrm{q} / \mathrm{ha} / \mathrm{an}$ chez les précoces, $0,6 \mathrm{chez}$ les demi-précoces et 0,8 chez les tardifs, soit, en moyenne, un gain légèrement supérieur à $0,8 \mathrm{q} / \mathrm{ha} / \mathrm{an}$. Parallèlement le pourcentage de verse diminue de 20,2 p. 100 pour les hybrides de 1950 à 3,5 p. 100 pour les hybrides de 1980 et le coefficient de régression du rendement par rapport à la densité passe de 0,08 à 0,59 (tabl. 9).

Le rendement national moyen a été durant les mêmes années (1982-1983-1984) de 62,2 q/ha par rapport à 18,9 q/ha pour la période 1952-1953-1954. Le gain annuel est de 1,44 q/ha/an. En chiffres bruts, la part due au progrès génétique est donc de 0,8 sur 1,44 soit 55 p. 100. En fait, la comparaison directe n'est pas possible puisque le rendement moyen des variétés récentes dans nos essais (environ $90 \mathrm{q} / \mathrm{ha}$ ) est audessus du rendement moyen en culture. Une possibilité serait d'ajuster le rendement moyen des essais au rendement agricole (RUSSELL, 1974), mais il faudrait tenir compte du reliquat de variétés anciennes en culture et surtout moduler en fonction de l'importance des groupes de précocité. Pour le groupe précoce, on peut calculer le rendement correspondant aux essais sur la base des rendements moyens départementaux, on obtient $70,6 \mathrm{q} /$ ha. En pondérant le progrès génétique ( $1 \mathrm{q} / \mathrm{ha} / \mathrm{an})$ de la moyenne en culture par rapport à la moyenne en essai ( 70 sur 90 ), la part du progrès génétique est de 54 p. 100 , qui semble être le chiffre à retenir et qui est d'ailleurs voisin du chiffre de 50 p. 100 généralement admis (Duvick, 1983 ; CAUDERON, 1983). Le même calcul est difficile à réaliser pour les groupes demi-précoce et tardif à cause de l'hétérogénéité des conditions de cultures due en particulier à l'irrigation.
Plusieurs tentatives ont été faites aux U.S.A. pour chiffrer le progrès génétique (DUVICK, 1984 ; RUSSELL, 1984 ; CASTLEBERRY et al., 1984). Les résultats mesurés par ces auteurs ou par des auteurs antérieurs cités par DuviCK (1984) montrent que le progrès sur 50 ans est de l'ordre de $1 \mathrm{q} / \mathrm{ha}$ /an et la part attribuée au progrès génétique varie de 33 p. 100 à 89 p. 100 .

Une partie du progrès génétique est due au passage des hybrides doubles aux hybrides trois voies et aux hybrides simples qui maximisent la valeur spécifique des combinaisons hybrides. La production de semences commerciales directement sur lignées suppose une excellente valeur propre des lignées et une technicité élevée en particulier en cas de différences importantes de précocité de floraison des lignées. Un essai sur la valeur propre des lignées a été mis en place en 1984 et 1985 pour quantifier la productivité des lignées et l'évolution de quelques caractères morphologiques et physiologiques. Il faut noter l'extraordinaire « longévité » de certaines lignées: F2 figurait dans I.N.R.A. 200 inscrit en 1957 et figure dans les hybrides les plus récents!

Les résultats de CASTLEBERRY et al. (1984) et de DUVICK (1984) semblent montrer des différences maximales en situation favorable alors que RUSSELL (1984) estime que le gain est supérieur en conditions difficiles. Les résultats que nous avons obtenus dans les groupes précoces et demi-précoces vont nettement dans le sens d'une meilleure régularité du rendement, c'est-à-dire que la différence entre les hybrides modernes et anciens est maximale dans les situations défavorables. On peut penser que cela résulte essentiellement de l'adaptation aux températures basses obtenues en particulier par l'introduction des maïs à grain corné (essentiellement la lignée F2) et peut être d'une meilleure tolérance à la sécheresse. Dans le groupe tardif, la situation est moins claire, entre 1950, 1960 et 1970, la sélection tend vers une meilleure adaptation aux conditions favorables, cette tendance se poursuit avec l'hybride prolifique MOHICAN. Par contre, dans ce groupe tardif, les autres hybrides de 1980 paraissent mieux adaptés aux environnements défavorables (fig. 3). CASTLEBERRY et al. (1984) montrent également, contrairement à une idée assez répandue, que même si les hybrides modernes répondent mieux à la fertilisation et à l'irrigation, ils produisent aussi davantage que les hybrides anciens en conditions sèches ou sans fertilisation.

TABLEAU 9

Résultats agronomiques par décennie (précocités confondues).

Agronomic results by decade (overall mean).

\begin{tabular}{|c|c|c|c|c|c|c|c|c|}
\hline \multirow{3}{*}{ Décennie } & \multicolumn{4}{|c|}{ Moyenne des densités } & \multicolumn{4}{|c|}{ Densité optimale } \\
\hline & \multicolumn{2}{|c|}{ Rendement } & \multirow{2}{*}{$\begin{array}{l}\% \text { eau à la } \\
\text { récolte }\end{array}$} & \multirow{2}{*}{$\%$ Verse } & \multicolumn{2}{|c|}{ Rendement } & \multirow{2}{*}{$\begin{array}{l}\text { Coefficient de } \\
\text { régression } \\
\text { rendement/ } \\
\text { densité }\end{array}$} & \multirow{2}{*}{$\begin{array}{c}\text { Index de } \\
\text { valeur } \\
\text { agronomique }\end{array}$} \\
\hline & q/ha & $\%$ & & & $q / h a$ & $\%$ & & \\
\hline 1950 & 73,2 & 100 & 26,9 & 20,2 & 74,6 & 100 & 0,08 & 100 \\
\hline 1960 & 82,9 & 113 & 28,2 & 12,4 & 86,8 & 116 & 0,31 & 118 \\
\hline 1970 & 88,1 & 120 & 27,1 & 7,2 & 92,3 & 124 & 0,32 & 130 \\
\hline 1980 & 91,7 & 125 & 26,7 & 3,5 & 98,6 & 132 & 0,59 & 144 \\
\hline
\end{tabular}


Un autre point de convergence est qu'une partie importante du progrès génétique observé est due à l'adaptation aux fortes densités, qui mettent en évidence les écarts maximaux entre variétés récentes et variétés anciennes. L'une des principales composantes de cette adaptation aux fortes densités est l'amélioration de la résistance à la verse qui est particulièrement nette dans nos essais. Il existe d'ailleurs une interaction permanente entre techniques culturales et variétés, l'évolution des variétés permet l'évolution des techniques culturales qui crée un nouveau milieu de sélection induisant un nouveau progrès génétique.

L'exemple du blé illustre parfaitement cette notion. Le progrès obtenu chez le blé pourrait se résumer à l'augmentation de la capacité de la culture à absorber l'azote et à la faculté, chez les variétés modernes, à conserver un épi fertile à des densités élevées (BOIZARD et LEFEVRE, résultats non publiés obtenus au CETA de HAM). Le désherbage chimique, la lutte contre les maladies (résistances génétiques, lutte chimique, précocité), les apports fractionnés d'azote et la résistance à la verse vont dans ce sens. L'amélioration de la résistance à la verse conduit à des variétés plus courtes et élève le rapport grain/paille (GRIGNAC, 1981). Chez le blé, le progrès paraît continu, même s'il semble un peu plus faible que chez le maïs ( $20 \mathrm{q} / \mathrm{ha}$ en 30 ans d'après CAUDERON, 1983). La production de grains s'accroît plus que la production de la plante entière (AUSTIN et al., 1980). Chez le blé également GRIGNAC (1981) souligne qu'en " conditions de culture sommaires ", c'est-à-dire à très faible niveau de rendement, les variétés anciennes, réputées plus souples, ne sont pas meilleures que les variétés récentes. Dans nos essais, seules les densités ont été prises en compte, toutes les techniques modernes ont été utilisées pour l'ensemble des hybrides : travail du sol, désherbage chimique..., la fertilisation n'est en principe jamais limitante.
Des mesures morphologiques : hauteur, surface de la feuille de l'épi, caractéristiques de l'épi, et physiologiques : vigueur au départ, date de floraison, ont été effectuées sur quelques essais. Elles devraient permettre d'aller plus loin dans l'analyse de l'élaboration du rendement. De même des essais «ensilage » permettront de chiffrer la productivité plante entière au stade ensilage et donc d'avoir une meilleure idée de l'évolution du rapport grain sur plante entière et plus généralement sur les transferts vers l'épi.

Toutes les données connues semblent montrer que le progrès génétique se poursuivra à moyen terme grâce à l'augmentation du potentiel des variétés et, peut être, surtout grâce à l'amélioration de la rusticité et donc de la régularité de rendement. L'amélioration continue de la résistance à la verse devrait permettre de poursuivre l'augmentation des densités de peuplement. La sélection est d'ailleurs déjà largement orientée vers cet objectif. Enfin, le potentiel augmentant, l'intensification des techniques culturales, devrait se poursuivre.

Ce travail a été réalisé en collaboration par le groupe maïs I.N.R.A. et par l'AGPM, avec la collaboration de J.-L. Algans, A. Baradat, C. Billot, A. Boyat, M. Caenen, M. Farnarie, L. Felix, F. KaAn, C. Maginieau, B. Mille, A. Panouille, M. Pollacsek, J.-P. SAmpouX.

\section{REMERCIEMENTS}

Nous remercions F. KAAN et P. GRIGNAC pour leur aide lors de la rédaction du manuscrit et $P$. SALON qui a réalisé un DEA sur les données obtenues dans ces essais.
Austin R. B., Bingham J., Blackwell R. D., Evans L. T., Morgan C. L., Taylor M., 1980. Genetic improvements in winter wheat yields since 1900 and associated physiological changes. J. Agric. Sci. Camb., 94, 675-689.

Bachacou J., Masson J.-P., Millier C., 1981. Amance 81, I.N.R.A.CNRF, $516 \mathrm{p}$.

Bloc D., Desvignes Ph., Gerbier N., Remois P., 1978. Contribution à l'étude agroclimatologique du maiss en France. Document Météorologie Nationale, AGPM et ITCF, $35 \mathrm{p}$.

Castleberry R. M., Crum C. W., Krull C. F., 1984. Genetic yield improvement of U.S. maize cultivars under varying fertility and climatic environments. Crop Sci., vol. 24, 33-36.

Cauderon A., 1958. Les mais hybrides en France. Etude de la précocité. Ann. Amélior. Plantes, 8 (3), 272-289.

Cauderon A., 1983. Techniques de production dans les systèmes de culture à base de céréales. Evolution en France: 1950-1980. Réflexions sur l'avenir. Cultivar, supplément au ${ }^{\circ} 158,23 \mathrm{p}$.
Derieux M., Darrigrand M., Gallais A., Barriere Y., Bloc D., Montalant Y., 1986. Estimation du progrès génétique réalisé chez le maïs grain en France entre 1950 et 1985. Rapport interne 32 p.

Duvick D. N., 1983. Les progrès de la sélection classique. Symposium de STARDLER, $12 \mathrm{p}$.

Duvick D. N., 1984. Genetic contributions to yield gains of U.S. hybrid maize, 1930 to 1980 , in « Genetic contributions to yield gains of five major crops plants ". W. R. Fehr ed., Crop Science Society of America special publication $n^{\circ} 7$, Madison Washington.

Grignac P., 1981. Comparaison de variétés anciennes et modernes de blé tendre à divers niveaux d'intensification dans un environnement méditerranéen. C.R. Acad. Agric. de France, 67, 1434-1453, Séance du 9 décembre 1981.

Russell W. A., 1974. Comparative performance of maize hybrids representing different eras of maize breeding. In " Proceedings of the 29th Annual Corn and Sorghum Research Conference ", Delores Wilkinson, ed., American Seed Trade Association, Washington, D.C.

Russell W. A., 1984. Agronomic performance of maize cultivars representing different eras of breeding. Maydica, XXIX, 375-390. 